\title{
Ligation, Transformation and Characterization of Rv 1926c Mycobacterium tuberculosis to Escherichia coli JM 109 For Latent Tuberculosis Immunodiagnostic
}

\author{
Rosana Agus ${ }^{1}$, Sjafaraenan ${ }^{1}$ and Mochammad Hatta ${ }^{2}$ \\ ${ }^{1}$ Department Of Biology, Faculty of Life Sciences, Hasanuddin University, Indonesia \\ ${ }^{2}$ Department of Microbiology, Faculty of Medicine, Hasanuddin University, Indonesia
}

\begin{abstract}
Tuberculosis caused by Mycobacterium tuberculosis is the biggest infectious disease causing human death in the world. The main challenge in controlling tuberculosis is to quickly and accurately diagnose tuberculosis infection. Several kits have been produced to diagnose tuberculosis, but have different sensitivity and specificity. This shows that the kit is not yet ideal for diagnosing tuberculosis, so the search for candidates for specific antigens still needs to be done. One potential antigen is the Rv 1926c encoding MPT 63 protein. This protein is known to induce Th1 cells and produce IFN $\lambda$ from PBMC cells of patients infected with tuberculosis. The purpose of this study was to clone the Rv 1926c from Mycobacterium tuberculosis as a tuberculosis immunodiagnostic kit. The method used is isolating Rv $1926 \mathrm{c}$ with PCR, ligation to PGEM-T vector and transformation to E.coli host cell JM 109. Clone characterization was carried out by PCR and migration analysis. The results obtained are the recombinant clones obtained have successfully inserted with the Rv 1926 c
\end{abstract}

\section{Article History}

Received 17 November 2018

Accepted 25 December 2018

Keyword

Rv 1926c

Immunodiagnostic

E.coli, JM 109

Latent

MPT 63

\section{Introduction}

Tuberculosis (TB) is the ninth leading cause of death worldwide. In 2016, there were an estimated 1.3 million TB deaths among HIV-negative people and an additional 374.000 deaths among HIV-positive people. In 2016 an estimated 10.4 million people fell ill with TB: $90 \%$ were adults, $65 \%$ were male, $10 \%$ were people living with HIV ( $74 \%$ in Africa) and $56 \%$ were in five countries: India, Indonesia, China, the Philippines and Pakistan (WHO, 2017).

Generally most of the people in Indonesia have received the BCG vaccine when they were toddlers. But the effectiveness of this vaccine does not last until adulthood, so it is suspected that everyone can be infected by $M$. tuberculosis and is latent. Latent TB infection 
has the potential to become active TB and people with active TB can be a source of new infections

Latent tuberculosis infection (LTBI) is the presence of tuberculosis in the body without symptoms or radiographic evidence or bacteriological examination. It is estimated that up to 13 million people in the United States are latent TB, and 5-10\% of infected people will suffer from TB, which is equivalent to 650,000 to $1,300,000$ (CDC, 2013).

The guidelines recommend that either Tuberculin Skin Test (TST) or Interferon gamma release assays (IGRA) can be use to test for latent TB in in high-income and upper middleincome countries with estimated TB incidence less than 100 per 100000 . Consistent with existing WHO recommendations, the guidelines reiterated that IGRA should not replace tuberculin skin test in low-income and other middle-income countries (WHO, 2018).

Tuberculin Skin Test (TST), also referred to as the Mantoux or Purified Protein Derivative (PPD) test, detects TB exposure through the skin. Advantages of TST are simple and easy to administer and can detect TB infection. Disadvantages of TST are false negatives: the failure of a bump to develop can be the result of a compromised immune system, false positives: the bacterial particles in the BCG vaccine can stimulate the production of TB antibodies, causing a false positive, sensitivity varies widely across populations and is inconclusive for children, HIV patients with low CD4 counts (TAG, 2018).

Some weaknesses of TST include not being able to distinguish between active and latent TB patients. This test will be positive in people who are BCG vaccinated and who are in contact with other mycobacteria (Pai et al., 2016). Therefore the specificity of PPD is questionable, especially in endemic areas such as Indonesia.

Due to the limitations of the TST test, the study was directed to find the specific antigen to be used as an immunodiagnostic. Especially the availability of TB diagnostic reagents that can identify new and latent infected individuals with high risk that can develop into active tuberculosis. One potential antigen is the Rv1926c encoding MPT 63 protein. This protein is known to induce Th1 cells and produce IFN $\lambda$ from PBMC cells of patients infected with TB.The purpose of this study was to clone the Rv 1926c from M. tuberculosis to E.coli JM 109 as immunodiagnostic latent tuberculosis

\section{Materials and Methods}

\section{Bacterial Strains and Plasmids}

The cloning vectors pGEM-T Easy and bacterial strain JM 109 (Promega) was used. The strains $M$. tuberculosis was obtained from clinical isolate from Indonesia.

\section{Culture Condition}

Bacterial strain JM 109 is a useful host for transformation of pGEM-T vector. Bacterial were incubate with stirring over night in Luria- Bertani medium in the presence of ampicillin $(1 \mu \mathrm{g} / \mathrm{ml})$ at $37^{\circ} \mathrm{C}$. The clinical isolate of $M$. tuberculosis is cultured in the Lowenstein-Jensen medium.

\section{Chromosome DNA extraction}

M.tuberculosis chromosome DNA extracted using Qiagen DNeasy kit. DNA extraction were measured using a spectrophotometer at absorbance of $260 \mathrm{~nm}$.

\section{Amplification}


Amplification of Rv 1926c was carried out by PCR using a specific primer. The primer sequence used in this study according (5) as follow Forward: 5'CAGCAGGATCCCGCCTATCCCATCACCGGA-3' and Reverse : 5'GCCCAAGCTTCGGCTCCCAAATCAGCAG-3' .The PCR conditions for amplification were pre denaturation $94^{\circ} \mathrm{C} 10$ minutes, denaturation $94^{\circ} \mathrm{C} 1$ minute, annealing $56^{\circ} \mathrm{C} 1$ minute and elongation $72^{\circ} \mathrm{C} 1$ minute in 30 cycles.

\section{Creation of Plasmid of the Recombinant Rv 1926c in E. coli JM 109}

Fragment of the Rv 1926c were obtained by PCR using two primers pair and DNA of the M. tuberculosis Indonesian strain as a template. The PGEM-T vector and PCR product were cut with BamHI and HindIII, mixed, and treated with T4 DNA ligase. The resulting recombinant plasmid pGEM-T-Rv1926 was transformed in E. coli JM 109.

\section{Transformation}

Isolation of DNA and transformation of the E. coli JM 109 cells were performed as described in the guidebook [6], with the following modifications. Transformed cells were spread on the appropriate indicator plates containing ampicillin. Colonies were scored for phenotype on Luria Bertani agar plates after 24 hours at $37^{\circ} \mathrm{C}$.

\section{Results and Discussion}

\section{PCR Product and Purification}

The results of PCR amplification of Rv 1926 observed by electrophoresis agarosa. A band was revealed which corresponded to the gene with the apparent of $412 \mathrm{bp}$.

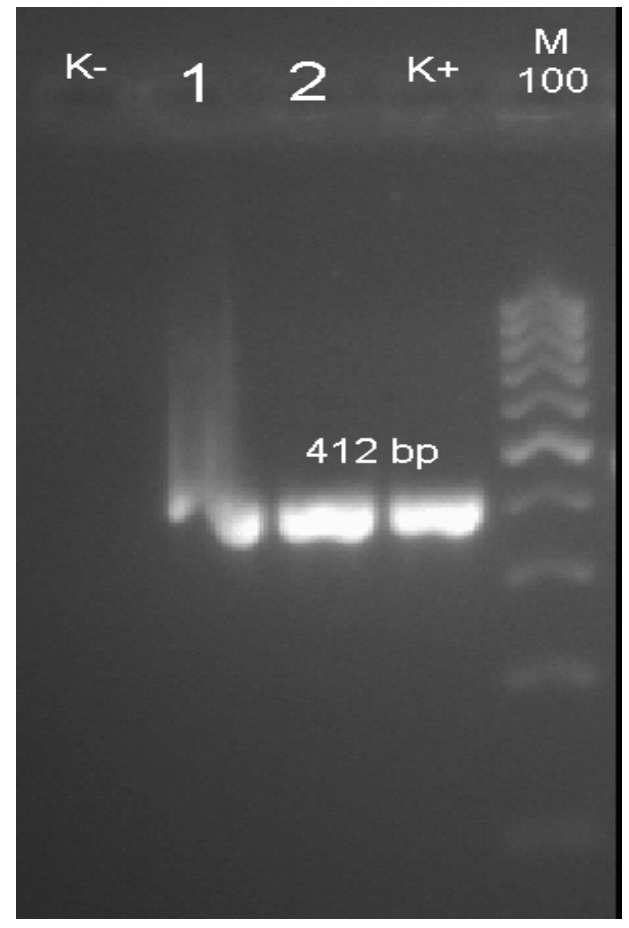

Figure 1. PCR product of Rv 1926c, (K-) Negative control , (1,2) Rv 1926c , (K+) Positive control , (M 100) Marker 100 bp 


\section{Ligation and transformation of the Rv 1926c to the pGEM-T vector}

The results of the transformation in Figure 1 showed that there were colonies and white colonies in petridish containing LB medium, X-gal, IPTG and ampicillin. White colonies indicate that the insertion DNA has been successfully inserted into the vector, while the blue colony means that the insertion DNA has not been successfully transferred to the vector. White $E$. coli colonies (transformant cells) showed Rv 1926c coding DNA successfully ligated in the MCS (multi cloning site) area found in the lacZ pGEM-T gene. The insertion of this DNA fragment will inhibit the lac $Z$ gene to encode the subunit of $\beta$-galactosidase, so that the enzyme cannot degrade the available galactose substrate. The bacterial colonies are blue, meaning they do not have insertion DNA fragments so they can degrade the available galactose substrate (Medical Biochemistry, 2017).

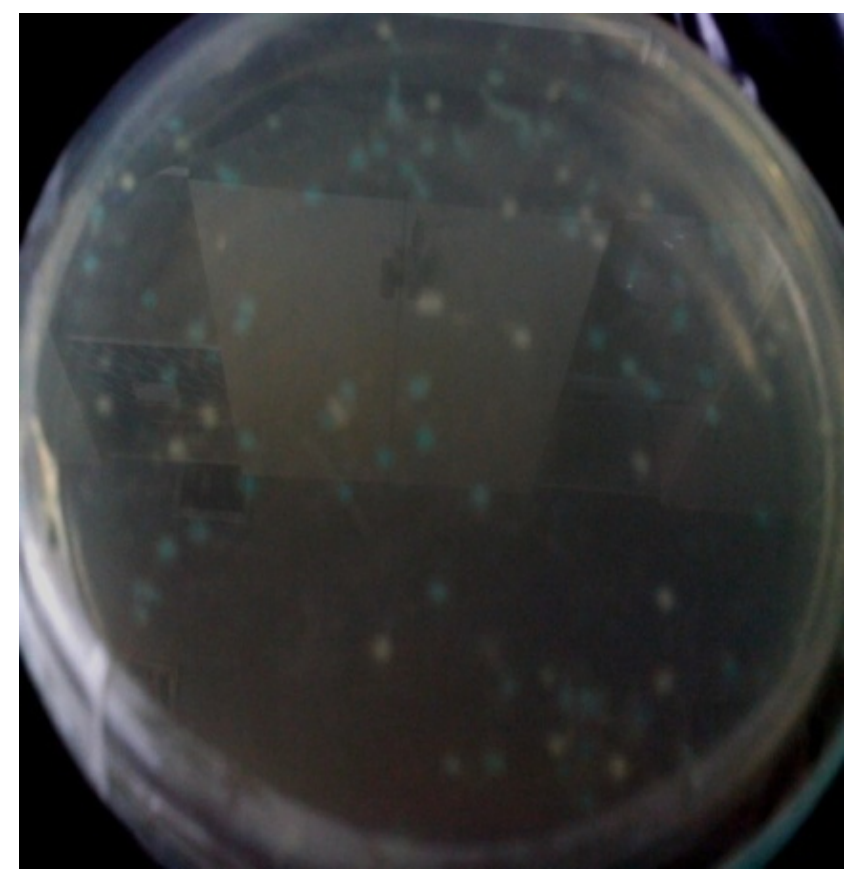

Figure 2. Recombinant clone pGEM-T-Rv 1926c

\section{Characterization of Recombinant Plasmid of pGEM-T-Rv 1926c}

Isolation of plasmid were performed according to the procedure instructions (BioRad). Characterization was done by PCR analysis and migration analysis. Migration analysis was done by comparing pGEM-T (3000 bp) and pGEM-T-Rv 1926c (3412 bp). A band shows difference length while pGEM-T-Rv1926c had slower migration than pGEM-T (Figure 3).

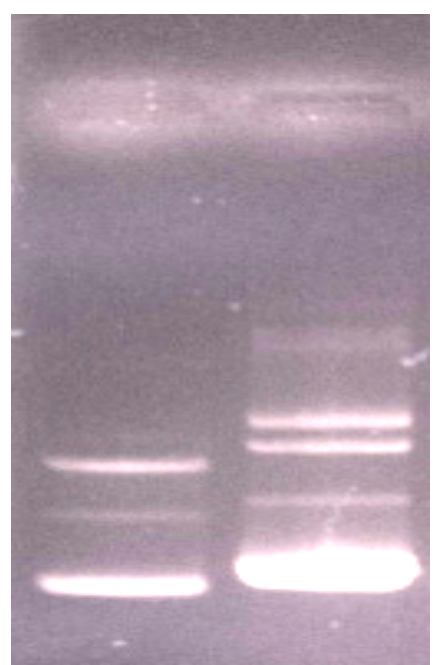


Figure 3. Migration analysis: (1) pGEM-T: 3000 bp (2) pGEM-T-Rv1926: 3412bp

This suggests that the plasmid without insert DNA (pGEM-T) will move faster than the recombinant plasmid (pGEM-T-Rv 1926c). This means that the DNA inserts Rv 1926c has been successfully ligation into the vector PGEM-T. PCR analysis was done by using exactly the same cycle as it mentioned before for amplified the Rv 1926c gene. Electrophoresis showed that plasmid recombinant contain the Rv 1926c gene as DNA insert was 412 bp (Figure 4)

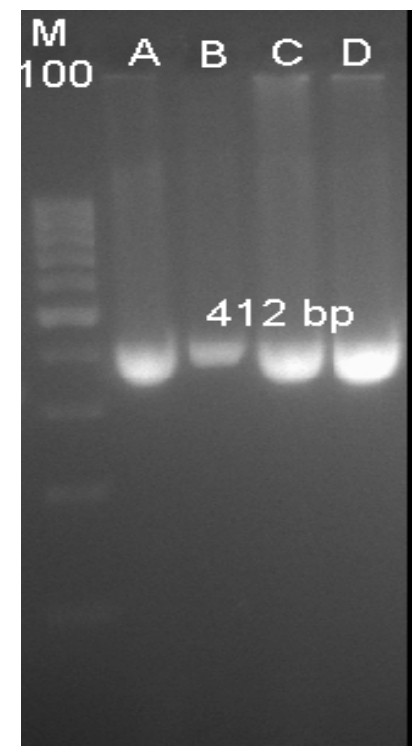

Figure 4. PCR analysis: (A,B,C,D) Rv 1926c : 412bp. (M) Marker 100 bp

\section{Conclusions}

Gene of Rv 1926c M. tuberculosis Indonesian isolates have been successfully ligated to $\mathrm{pGEM}-\mathrm{T}$ vectors and transformed into E. coli JM 109

\section{Acknowledgments}

The authors would like to thank Kemenristek Dikti which has funded this research through a PDUPT 2018

\section{References}

Centers for Disease Control and Prevention National, 2013, Health, United States, With Special Feature on Prescription Drug

Medical Biochemistry, 2017, Blue White Screening

Pai, M. and Giovanni Sotgiu, 2016, Diagnostics for latent TB infection: incremental, not transformative progress, Eur Respir J 2016; 47: 704-706 |

Samal, 2012, Evaluation of diagnostic potential of recombinant MPT63 protein in bovine tuberculosis, Deemed University Indian Veterinary Research Institute

Treatment Action Group, 2018, Module Four TB Diagnostics, TB/HIV Advocacy Toolkit 
World Health Organization, 2017, Global tuberculosis report

World Health Organization, 2018, WHO guidelines on the management of latent tuberculosis infection launched today

Sambrook, J., Fristch, E.F. dan Maniatis, T. Molecular Cloning A Laboratory Manual. Cold Spring Harbor Laboratory Press, 1989.

Treatment Action Group, 2018, Module Four TB Diagnostics, TB/HIV Advocacy Toolkit 